\title{
The Algebra Generated by Physical Filters *
}

\author{
Günter Dähn \\ Mathematisches Institut der Universität Tübingen
}

Received January 31, 1972

\begin{abstract}
This paper investigates mathematical properties of a finite-dimensional real algebra of linear operators which are generated by an orthomodular lattice of filters in the sense of Mielnik [4]. Properties of filter decomposability and a representation theorem for the vector space underlying the algebra mentioned are derived.
\end{abstract}

\section{Introduction}

The physical background and the motivation of the subsequent mathematical investigations are the papers by Ludwig [3] whose axiom system was, together with the most important mathematical consequences, restated in [2] in a way more adapt to our mathematical considerations. So, referring to [2] for detailed mathematical notes, we will here only sketch basic mathematical concepts in a contemporary language.

A comprehensive and careful analysis of all current attempts of an axiomatic foundation of physical theories has been given by Mielnik [4] who has subordinated the lattice-representing operators $\boldsymbol{T}_{E}$ of [2] to the physical concept of filters.

\section{Preliminaries}

We start from a dual pair $\left(B, B^{\prime}\right)$ of two real topological vector spaces. As in [2] $B$ (and hence $B^{\prime}$ ) are supposed to be finite-dimensional, say $\operatorname{dim} B=\operatorname{dim} B^{\prime}=N$.

1. $B$ has an order base $K$ which is convex and closed. The elements of $K$ are denoted by $V$, the elements of $B$ in general by $X$.

2. In $B$ there exists a proper positive generating cone $B_{+}$generated by $K$, i.e.

$$
B=B_{+}-B_{+}, B_{+}=\bigcup_{\lambda \in \boldsymbol{R}_{+}} \lambda K
$$

3. $B^{\prime}$ is partially ordered by

$$
Y_{1} \leqq Y_{2}: \Leftrightarrow\left\langle V, Y_{1}\right\rangle \leqq\left\langle V, Y_{2}\right\rangle \text { for every } V \in K .
$$

* This paper leans on a report presented to and supported by the Deutsche Forschungsgemeinschaft. 
4. $B^{\prime}$ has an order unit $\mathbf{1}$ with $L:=\{Y \mid 0 \leqq Y \leqq \mathbf{1}\}$. $L$ is convex and closed. Its elements are denoted by $F .\langle V, \mathbf{1}\rangle=1$ for every $V \in K$. (In [2] $L$ was denoted by $\hat{L}$.)

5. $B^{\prime}$ is generated by a proper positive cone $B_{+}^{\prime}$ generated by $L$, i.e.

$$
B^{\prime}=B_{+}^{\prime}-B_{+}^{\prime}, B_{+}^{\prime}=\bigcup_{\lambda \in \boldsymbol{R}_{+}} \lambda L .
$$

6. The canonical bilinear functional $\langle\cdot, \cdot\rangle$ over $B \times B^{\prime}$ is the extension of the (physical motivated) function $\mu$ over $K \times L$ restricted by

$$
0 \leqq \mu(V, F) \leqq 1 \text { for all }(V, F) \in K \times L
$$

separates points in $K$ and $L$ respectively.

7. $B$ is a real Banach space by

$$
\|X\|:=\sup \{\mid \mu(X, F) \| F \in L\} \text { for each } X \in B .
$$

$B^{\prime}$ is the dual Banach space by

$$
\|Y\|:=\sup \{|\mu(X, Y)| \mid X \in B \text { and }\|X\|=1\} \text { for each } Y \in B^{\prime} .
$$

8. The extreme points $E$ of $L$ form an orthomodular lattice $G$ with 0 and $\mathbf{1}$ as zero and unit element, respectively. For every $E \in G$ the orthocomplement $E^{\perp}$ of $E$ is defined by $E^{\perp}=\mathbf{1}-E$.

$A(G)$ denotes the set of all atoms $P$ of $G, A(W)$ the set of all atoms of $W$ (see 10.).

9. With the notations

$$
\begin{array}{lll}
K_{i}(l):=\{V \mid\langle V, F\rangle=i & \text { for all } F \in l\}, i=0 ; 1 \quad \text { and any } \quad l \cong L, \\
L_{i}(k):=\{F \mid\langle V, F\rangle=i & \text { for all } V \in k\}, i=0 ; 1 \text { and any } k \cong K
\end{array}
$$

there exists a lattice isomorphism between $G$ and $U:=\left\{L_{0}(k) \mid k \leqq K\right\}$ and a dual lattice isomorphism between $G$ and $W:=\left\{K_{0}(l) \mid l \leqq L\right\}$.

10. $W$ is the set of all extremal sets of $K$ and equals the set of all facets of $K$ ([2], Theorem 2 and corollaries).

11. Throughout this paper $N_{n}$ denotes the interval $[1 ; n] \cap \boldsymbol{N}$ for any $n \in N$.

\section{Further Properties of the Operators $T_{E}$}

As in [2] $\mathscr{B}\left(B^{\prime}\right)$ denotes the $\boldsymbol{R}$-algebra of all linear (bounded) operators over $B^{\prime}$.

$$
\mathscr{T}:=\left\{\boldsymbol{T} \mid \boldsymbol{T} \in \mathscr{B}\left(B^{\prime}\right) \text { and } \boldsymbol{T}\left[B_{+}^{\prime}\right] \cong B_{+}^{\prime}\right\}
$$

is a proper positive cone in $\mathscr{B}\left(B^{\prime}\right)([2])$. There we defined

$$
\mathscr{T}(G):=\left\{\boldsymbol{T}_{E} \mid \boldsymbol{T}_{E} \in \mathscr{T} \quad \text { and } \quad E \in G\right\}
$$


with $\boldsymbol{T}_{E}$ uniquely determined by $\left\langle V, \boldsymbol{T}_{E} F\right\rangle=\langle V, F\rangle$ for all $V \in K_{1}(E)$. $T_{E}$ was proved to be idempotent and $\mathscr{T}(G)$ was shown to be an orthomodular lattice isomorphic to $G$.

This section is mainly devoted to the answer of two open questions at the end of [2] (questions 4 and 5). Results similar to those concerning perpendicular projectors on Hilbert space will be obtained.

Theorem 1. For all $\boldsymbol{T}_{E_{1}}, \boldsymbol{T}_{E_{2}} \in \mathscr{T}(G): \boldsymbol{T}_{E_{1} \wedge E_{2}}=\boldsymbol{T}_{E_{1}} \boldsymbol{T}_{E_{2}}$ iff $\boldsymbol{T}_{E_{1}} \boldsymbol{T}_{E_{2}}=\boldsymbol{T}_{E_{2}} \boldsymbol{T}_{E_{1}}$.

Proof. (i) If $\boldsymbol{T}_{E_{1} \wedge E_{2}}=\boldsymbol{T}_{E_{1}} \boldsymbol{T}_{E_{2}}$, then $\boldsymbol{T}_{E_{1}} \boldsymbol{T}_{E_{2}}=\boldsymbol{T}_{E_{2}} \boldsymbol{T}_{E_{1}}$ because of $\boldsymbol{T}_{E_{1} \wedge E_{2}}=\boldsymbol{T}_{E_{2} \wedge E_{1}}$.

(ii) Suppose $\boldsymbol{T}_{E_{1}} \boldsymbol{T}_{E_{2}}=\boldsymbol{T}_{E_{2}} \boldsymbol{T}_{E_{1}}$. Since $\boldsymbol{T}_{E_{1}}(i=1 ; 2)$ are idempotent, so is $\boldsymbol{T}_{E_{1}} \boldsymbol{T}_{E_{2}}$. According to the isomorphism Theorem 16 from [2] there holds $\boldsymbol{T}_{E_{1} \wedge E_{2}}=\boldsymbol{T}_{E_{1}} \wedge \boldsymbol{T}_{E_{2}}$; thus, on the one hand, $\left\langle V, \boldsymbol{T}_{E_{1} \wedge E_{2}} F\right\rangle=\langle V, F\rangle$ for all $V \in K_{1}\left(E_{1} \wedge E_{2}\right)=K_{1}\left(E_{1}\right) \cap K_{1}\left(E_{2}\right)$ and, on the other hand, $\left\langle V, \boldsymbol{T}_{E_{1}} \boldsymbol{T}_{E_{2}} F\right\rangle=\left\langle V, \boldsymbol{T}_{E_{2}} \boldsymbol{T}_{E_{1}} F\right\rangle$ for all $V \in K$. So there holds especially: $\left\langle V, \boldsymbol{T}_{E_{1}} \boldsymbol{T}_{E_{2}} F\right\rangle=\left\langle V, \boldsymbol{T}_{E_{2}} F\right\rangle$ for all $V \in K_{1}\left(E_{1}\right)$ and $\left\langle V, \boldsymbol{T}_{E_{2}} \boldsymbol{T}_{E_{1}} F\right\rangle=\left\langle V, \boldsymbol{T}_{E_{1}} F\right\rangle$ for all $V \in K_{1}\left(E_{2}\right)$.

Therefore $\left\langle V, \boldsymbol{T}_{E_{1}} \boldsymbol{T}_{E_{2}} F\right\rangle=\langle V, F\rangle$ holds for all $V \in K_{1}\left(E_{1} \wedge E_{2}\right)$, i.e. $\left\langle V, \boldsymbol{T}_{E_{1}} \boldsymbol{T}_{E_{2}} F\right\rangle=\left\langle V, \boldsymbol{T}_{E_{1} \wedge E_{2}} F\right\rangle$ for all $V \in K_{1}\left(E_{1} \wedge E_{2}\right)$. This is, according to Theorem 9 in [2], sufficient for $\boldsymbol{T}_{E_{1}} \boldsymbol{T}_{E_{2}} F=\boldsymbol{T}_{E_{1} \wedge E_{2}} F$ for all $F \in L$ because of $\boldsymbol{T}_{E_{1}} \boldsymbol{T}_{E_{2}} F \leqq E_{1} \wedge E_{2}$ by hypothesis. Hence we obtain $\boldsymbol{T}_{E_{1}} \boldsymbol{T}_{E_{2}}=\boldsymbol{T}_{E_{1} \wedge E_{2}}$.

Corollary. Let $\tau$ be any finite subset of $\mathscr{T}(G): \bigwedge_{\tau} T_{E}=\prod_{\tau} T_{E}$ iff $\tau$ consists of pairwise commuting elements.

Proof. By induction.

In [2] we considered the Sasaki-projection $\Phi_{e}$ defined by $\Phi_{e}(g)$ $=e \wedge\left(g \vee e^{\perp}\right)$ for all $g$ of an orthomodular lattice and any $e$ therein. This projection $\Phi_{e}$ was compared with the projector $\boldsymbol{T}_{E}$. With the compatibility relation " $e_{1} \mathscr{C} e_{2}$ iff $e_{1}=\left(e_{1} \wedge e_{2}\right) \vee\left(e_{1} \wedge e_{2}^{\frac{1}{2}}\right)$ " there holds due to Nakamura [5] in any orthomodular lattice " $e_{1} \mathscr{C} e_{2}$ iff $\Phi_{e_{1}} \Phi_{e_{2}}$ $=\Phi_{e_{2}} \Phi_{e_{1}}{ }$.

Concerning $\boldsymbol{T}_{E}$ the validity of this equivalence was the open question 4 in [2]. The next theorem answers this question in the affirmative.

Theorem 2. For all $E_{1}, E_{2} \in G: E_{1} \mathscr{C} E_{2}$ iff $\boldsymbol{T}_{E_{1}} \boldsymbol{T}_{E_{2}}=\boldsymbol{T}_{E_{2}} \boldsymbol{T}_{E_{1}}$.

Proof. (i) Given $E_{1}, E_{2} \in G$ such that $E_{1} \mathscr{C} E_{2}$ is valid, i.e. $E_{1}$ $=\left(E_{1} \wedge E_{2}\right) \stackrel{\Perp}{ }\left(E_{1} \wedge E_{2}^{\perp}\right)$. Then $\quad \boldsymbol{T}_{E_{1}} F=\boldsymbol{T}_{\left(E_{1} \wedge E_{2}\right)+\left(E_{1} \wedge E_{2}\right)} F \leqq E_{1} \wedge E_{2}$ $+E_{1} \wedge E_{2}^{\perp}$, hence $\boldsymbol{T}_{E_{2}} \boldsymbol{T}_{E_{1}} F \leqq E_{1} \wedge E_{2}$ for all $F \in L$. Since $\boldsymbol{T}_{E_{1} \wedge E_{2}} \boldsymbol{T}_{E_{2}} \boldsymbol{T}_{E_{1}}$ $=\boldsymbol{T}_{E_{1} \wedge E_{2}}$, we conclude from the definition of the $\boldsymbol{T}_{E}$-operator $\left\langle V, \boldsymbol{T}_{E_{1} \wedge E_{2}} \boldsymbol{T}_{E_{2}} \boldsymbol{T}_{E_{1}} F\right\rangle=\left\langle V, \boldsymbol{T}_{E_{2}} \boldsymbol{T}_{E_{1}} F\right\rangle=\left\langle V, \boldsymbol{T}_{E_{1} \wedge E_{2}} F\right\rangle=\langle V, F\rangle$ for all $V \in K_{1}\left(E_{1} \wedge E_{2}\right)$ and any $F \in L$. Then Theorem 9 of [2] gives, because of $\boldsymbol{T}_{E_{2}} \boldsymbol{T}_{E_{1}} F \leqq E_{1} \wedge E_{2}, \boldsymbol{T}_{E_{1} \wedge E_{2}} F=\boldsymbol{T}_{E_{2}} \boldsymbol{T}_{E_{1}} F$ for all $F \in L$, thus $\boldsymbol{T}_{E_{1}} \boldsymbol{T}_{E_{2}}=\boldsymbol{T}_{E_{2}} \boldsymbol{T}_{E_{1}}$ by Theorem 1 . 
(ii) Supposing $\boldsymbol{T}_{E_{1}} \boldsymbol{T}_{E_{2}}=\boldsymbol{T}_{E_{2}} \boldsymbol{T}_{E_{1}}$, we obtain $\boldsymbol{T}_{E_{1}} \boldsymbol{T}_{E_{2}}=\boldsymbol{T}_{E_{1} \wedge E_{2}}$ by Theorem 1. Since $E_{1} \wedge E_{2} \leqq E_{1}$ orthomodularity of $G$ implies $E_{1}=\left(E_{1} \wedge E_{2}\right) \triangleright\left(E_{1} \wedge\left(E_{1} \wedge E_{2}\right)^{\perp}\right)$. To prove the assertion means to prove $E_{1} \wedge\left(E_{1} \wedge E_{2}\right)^{\perp}=E_{1} \wedge E_{2}^{\perp}$. This will have been verified when $E_{1} \wedge\left(E_{1} \wedge E_{2}\right)^{\perp} \leqq E_{1} \wedge E_{2}^{\perp}$ has been verified because $E_{1} \wedge\left(E_{1} \wedge E_{2}\right)^{\perp}$ $\geqq E_{1} \wedge E_{2}^{\perp}$ holds always. To this end we observe that $\boldsymbol{T}_{E_{1}} \boldsymbol{T}_{E_{2}}=\boldsymbol{T}_{E_{1} \wedge E_{2}}$ implies $\boldsymbol{T}_{E_{2}} \boldsymbol{T}_{E_{1}} \boldsymbol{T}_{\left(E_{1} \wedge E_{2}\right)^{+}}=\mathbf{0}$. This means $\left\langle V, \boldsymbol{T}_{E_{2}} \boldsymbol{T}_{E_{1}} \boldsymbol{T}_{\left(E_{1 \wedge} \wedge E_{2}\right)^{+}} F\right\rangle=0$ for all $V \in K$ and any $F \in L$; thus, in particular $\left\langle V, \boldsymbol{T}_{E_{2}} \boldsymbol{T}_{E_{1}} \boldsymbol{T}_{\left(E_{1} \wedge E_{2}\right)^{+}} F\right\rangle$ $=\left\langle V, \boldsymbol{T}_{E_{1}} \boldsymbol{T}_{\left(E_{1} \wedge E_{2}\right)^{\lrcorner}} F\right\rangle=0$ for all $V \in K_{1}\left(E_{2}\right)$ and any $F \in L$. This implies $\boldsymbol{T}_{E_{1}} \boldsymbol{T}_{\left(E_{1} \wedge E_{2}\right)^{\perp}} F \in L_{0} K_{1}\left(E_{2}\right)=L_{0} K_{0}\left(E_{2}^{\perp}\right)$ for all $F \in L$, hence $\boldsymbol{T}_{E_{1}} \boldsymbol{T}_{\left(E_{1} \wedge E_{2}\right)^{\prime}} F$ $\leqq E_{2}^{\perp}$ for all $F \in L$.

Consequently there holds especially $\boldsymbol{T}_{E_{1}} \boldsymbol{T}_{\left(E_{1} \wedge E_{2}\right)^{\perp}} E_{1} \wedge\left(E_{1} \wedge E_{2}\right)^{\perp}$ $=E_{1} \wedge\left(E_{1} \wedge E_{2}\right)^{\perp} \leqq E_{2}^{\perp}$. This yields $E_{1} \wedge\left(E_{1} \wedge E_{2}\right)^{\perp} \leqq E_{1} \wedge E_{2}^{\perp}$ and so $E_{1} \mathscr{C} E_{2}$.

Definition 1 ([6]). (i) Two idempotents $\boldsymbol{I}_{1}, \boldsymbol{I}_{2} \in \mathscr{B}\left(B^{\prime}\right)$ are said to be orthogonal iff $\boldsymbol{I}_{1} \boldsymbol{I}_{2}=\boldsymbol{I}_{2} \boldsymbol{I}_{1}=\mathbf{0}$.

(ii) An idempotent $\boldsymbol{I} \in \mathscr{B}\left(B^{\prime}\right)$ is called primitive, irreducible or minimal iff it cannot be decomposed into a sum of two orthogonal idempotents of $\mathscr{B}\left(B^{\prime}\right)$.

Next it will be shown that the orthogonality relation from the preceding definition if restricted to $\mathscr{T}(G)$ is equivalent with the latticetheoretical one defined in Theorem 16 of [2].

Theorem 3. For all $\boldsymbol{T}_{E_{1}}, \boldsymbol{T}_{E_{2}} \in \mathscr{T}(G): \boldsymbol{T}_{E_{1}} \leqq\left(\boldsymbol{T}_{E_{2}}\right)^{\perp}$ iff $\boldsymbol{T}_{E_{1}} \boldsymbol{T}_{E_{2}}=\boldsymbol{T}_{E_{2}} \boldsymbol{T}_{E_{1}}=\mathbf{0}$.

Proof. (i) Suppose $\boldsymbol{T}_{E_{1}} \leqq\left(\boldsymbol{T}_{E_{2}}\right)^{\perp}=\boldsymbol{T}_{E_{2}}$, i.e. $\boldsymbol{T}_{E_{1}}=\boldsymbol{T}_{E_{2}} \boldsymbol{T}_{E_{1}}=\boldsymbol{T}_{E_{1}} \boldsymbol{T}_{E_{2}}$. By Corollary 1 to Theorem 13 in [2] $\boldsymbol{T}_{E} \boldsymbol{T}_{E^{ \pm}}=\mathbf{0}$ for all $E \in G$. Therefore $\boldsymbol{T}_{E_{2}} \boldsymbol{T}_{E_{1}}=\boldsymbol{T}_{E_{2}} \boldsymbol{T}_{E_{2}} \boldsymbol{T}_{E_{1}}=\mathbf{0}$ and $\boldsymbol{T}_{E_{1}} \boldsymbol{T}_{E_{2}}=\boldsymbol{T}_{E_{1}} \boldsymbol{T}_{E_{2}} \boldsymbol{T}_{E_{2}}=\mathbf{0}$.

(ii) Supposing $\boldsymbol{T}_{E_{1}} \boldsymbol{T}_{E_{2}}=\boldsymbol{T}_{E_{2}} \boldsymbol{T}_{E_{1}}=\mathbf{0}$, we have $\boldsymbol{T}_{E_{1}} \boldsymbol{T}_{E_{2}} E_{2}=\boldsymbol{T}_{E_{1}} E_{2}=0$, i.e. $\left\langle V, \boldsymbol{T}_{E_{1}} E_{2}\right\rangle=\left\langle V, E_{2}\right\rangle=0$ for all $V \in K_{1}\left(E_{1}\right)$, thus $K_{1}\left(E_{1}\right) \leqq K_{0}\left(E_{2}\right)$ $=K_{1}\left(E_{2}^{\perp}\right)$, hence $E_{1} \leqq E_{2}^{\perp}$. Then the isomorphism Theorem 16 of [2] yields $\boldsymbol{T}_{E_{1}} \leqq\left(\boldsymbol{T}_{E_{2}}\right)^{\perp}$.

Theorem 17 in [2] expresses that $\boldsymbol{T}_{E_{1}} \lesseqgtr \boldsymbol{T}_{E_{2}}$ implies $\boldsymbol{T}_{E_{1}} \underset{\mathscr{T}(G)}{\subseteq} \boldsymbol{T}_{E_{2}}$. The converse of this theorem was formulated as an open question in [2] (question 5) the answer of which shall now be given.

Lemma 1. For all $\boldsymbol{T}_{E} \in \mathscr{T}(G)$ : if $\boldsymbol{T}_{E}$ is orthoadditively decomposable into $\boldsymbol{T}_{E}=\boldsymbol{T}_{E_{1}}+\boldsymbol{T}_{E_{2}}$, then $\boldsymbol{T}_{E}=\boldsymbol{T}_{E_{1} \vee E_{2}}$.

Proof. As a consequence of $E_{1} \perp E_{2}: \boldsymbol{T}_{E} \boldsymbol{T}_{E_{1}+E_{2}}=\left(\boldsymbol{T}_{E_{1}}+\boldsymbol{T}_{E_{2}}\right) \boldsymbol{T}_{E_{1}+E_{2}}$ $=\boldsymbol{T}_{E_{1}} \boldsymbol{T}_{E_{1}+E_{2}}+\boldsymbol{T}_{E_{2}} \boldsymbol{T}_{E_{1}+E_{2}}=\boldsymbol{T}_{E_{1}}+\boldsymbol{T}_{E_{2}}=\boldsymbol{T}_{E}$, thus $\boldsymbol{T}_{E} \leqq \boldsymbol{T}_{E_{1}+E_{2}}$. Besides, $\boldsymbol{T}_{E}\left(E_{1}+E_{2}\right)=\boldsymbol{T}_{E_{1}}\left(E_{1}+E_{2}\right)+\boldsymbol{T}_{E_{2}}\left(E_{1}+E_{2}\right)=E_{1}+E_{2} \leqq E$, thus $\boldsymbol{T}_{E_{1}+E_{2}}$ $\leqq T_{E}$. 

Boolean.

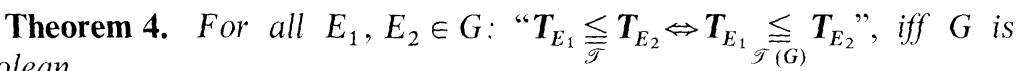

Proof. The implication " $\boldsymbol{T}_{E_{1}} \subseteq \boldsymbol{T}_{E_{2}} \Rightarrow \boldsymbol{T}_{E_{1}} \subseteq \boldsymbol{T}_{E_{2}}$ " holds by Theorem 17 of [2] in any orthomodular lattice $G$. Therefore the converse must be shown.

(i) Let $G$ be Boolean. $\boldsymbol{T}_{E_{1}} \underset{g(G)}{\leqq} \boldsymbol{T}_{E_{2}}$ implies $E_{1} \leqq E_{2}$ and so $E_{2}=E_{1} \vee\left(E_{2} \wedge E_{1}^{\perp}\right) . G$ being Boolean, Theorem 18 of [2] gives $\boldsymbol{T}_{E_{1}+\left(E_{2} \wedge E_{1}\right)}=\boldsymbol{T}_{E_{1}}+\boldsymbol{T}_{E_{2} \wedge E_{1}}=\boldsymbol{T}_{E_{2}}$, thus $\boldsymbol{T}_{E_{2}}-\boldsymbol{T}_{E_{1}}=\boldsymbol{T}_{E_{2} \wedge E_{4}} \frac{\geqq}{\bar{T}}$, i.e. $\boldsymbol{T}_{E_{2}} \gtreqless \boldsymbol{T}_{E_{1}}$.

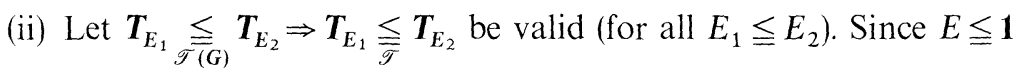

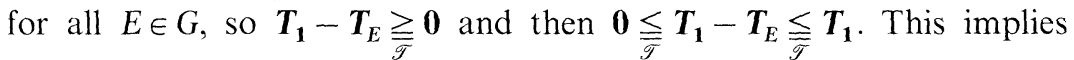
$\left(\boldsymbol{T}_{\mathbf{1}}-\boldsymbol{T}_{E}\right) F \in L$ for all $F \in L$; because of $\left\langle V,\left(\boldsymbol{T}_{\mathbf{1}}-\boldsymbol{T}_{E}\right) F\right\rangle=0$ for all $V \in K_{1}(E),\left(\boldsymbol{T}_{\mathbf{1}}-\boldsymbol{T}_{E}\right) F \in L_{0} K_{1}(E)=L_{0} K_{0}\left(E^{\perp}\right)$. Hence $\left(\boldsymbol{T}_{\mathbf{1}}-\boldsymbol{T}_{E}\right) F \leqq E^{\perp}$ for all $F \in L$. Consequently, each $F \in L$ is reduced by any $E \in G$ and from the proof of Theorem 18 in [2] there follows that $G$ is Boolean.

Theorem 5. If $\boldsymbol{T}_{E_{0}} \in \mathscr{T}(G)$ is orthodecomposable, then the segment $G\left(0, E_{0}\right)$ is a reducible lattice.

Proof. $G\left(0, E_{0}\right)$ is orthomodular with $E_{0} \wedge E^{\perp}$ as the orthocomplement for any $E \leqq E_{0}$. By hypothesis, $\boldsymbol{T}_{E_{0}}=\boldsymbol{T}_{E_{1}}+\boldsymbol{T}_{E_{2}}$. Lemma 1 implies $E_{0}=E_{1}$ $+E_{2}=E_{1}+E_{0} \wedge E_{1}^{\perp}$. Then, according to Theorem 4, each $F \in L_{E_{0}}$ is reduced by $E_{1}$ and $E_{0} \wedge E_{1}^{\perp}$, which, therefore, belong to the center of $G\left(0, E_{0}\right)$ thus being reducible.

Corollary. If $G$ is irreducible, then there exists no proper orthodecomposition of $\boldsymbol{T}_{\mathbf{1}}$.

Remark 1. Given the hypothesis of Theorem 5, there holds for every $E \in G\left(0, E_{0}\right)$ :

$$
E=\left(E \wedge E_{1}\right) \vee\left(E \wedge E_{0} \wedge E_{1}^{\perp}\right)=\left(E \wedge E_{1}\right) \forall\left(E \wedge E_{1}^{\perp}\right),
$$

which means that also in $G E \mathscr{C} E_{1}$ holds for all $E \in G\left(0, E_{0}\right)$. Now we wish to investigate when all segments $G(0, E)$ of the orthomodular lattice $G$ are irreducible, i.e. when no $\boldsymbol{T}_{E} \in \mathscr{T}(G)$ is orthodecomposable. We first illustrate this situation by the example of Hilbert space from Remark 4 in [2] thereby correcting it:

Let $\mathscr{H}$ be a finite-dimensional real Hilbert space and $G$ the lattice of all perpendicular projectors on $\mathscr{H} . L$ is then the set of all self-adjoint operators $F$ with $0 \leqq F \leqq 1 . T_{E}$ is given by $\boldsymbol{T}_{E} F=E F E$ for any $E \in G$ and all $F \in L . G$ is modular and we suppose it to be irreducible. Assume the existence of $E \in G$ such that $E F E=\left(E_{1}+E_{2}\right) F\left(E_{1}+E_{2}\right)=E_{1} F E_{1}$ 
$+E_{2} F E_{2}$ with $E_{1} \perp E_{2} \quad E_{1} F E_{2}$ being positive, we obtain $E_{1} F E_{2}$ $=E_{2} F E_{1}=0$. Every $F \in L$ has a decomposition $F=\sum_{i \in \boldsymbol{N}_{n}} \lambda_{i}^{F} P_{i}^{F}$ with pairwise orthogonal atoms $P_{i}^{F}$ and $\lambda_{i}^{F} \in \boldsymbol{R}_{+}^{*}$. Let $E_{1}=\sum_{j \in \boldsymbol{N}_{m}} P_{j}^{1}, E_{2}=\sum_{k \in \boldsymbol{N}_{l}} P_{k}^{2}$ be atomic orthodecompositions. Then $\sum_{j \in \boldsymbol{N}_{m}} P_{j}^{1} \sum_{i \in \boldsymbol{N}_{n}} \lambda_{i}^{F} P_{i}^{F} \sum_{k \in \boldsymbol{N}_{l}} P_{k}^{2}=0 \mathrm{im}-$ plies, again by positivity, $P_{j}^{1} P_{i} P_{k}^{2}=0$ for all $i \in N_{n}, j \in N_{m}, k \in N_{l}$. G being irreducible, the last equation cannot be valid for all $F \in L$ : let us consider $x_{j} \in \operatorname{Im} P_{j}^{1}, y_{k} \in \operatorname{Im} P_{k}^{2}, z_{i} \in \operatorname{Im} P_{i}^{F}$. Since $P_{j} P_{k}=0$, so $x_{j} \perp y_{k} . P_{x_{j}} P_{z_{i}} P_{y_{k}} h=0$ for all $h \in \mathscr{H}$ implies $\left\langle x_{j} \mid z_{i}\right\rangle\left\langle z_{i} \mid y_{k}\right\rangle\left\langle y_{k} \mid h\right\rangle=0$ for all $h \in \mathscr{H}$, hence $\left\langle x_{j} \mid z_{i}\right\rangle=0$ or $\left\langle z_{i} \mid y_{k}\right\rangle=0$. This contradicts the fact that in the 2-dimensional subspace generated by $x_{j}, y_{k}$ not all vectors $z$ are orthogonal to $x_{j}$ and $y_{k}$, respectively. This statement should be inferred from our general frame.

Theorem 6. If $G$ is modular and irreducible, no $\boldsymbol{T}_{E} \in \mathscr{T}(G)$ is nontrivially orthodecomposable in $\mathscr{T}(G)$.

Proof. Assume the existence of $\boldsymbol{T}_{E} \neq \boldsymbol{T}_{\mathbf{1}}$ with $\boldsymbol{T}_{E}=\boldsymbol{T}_{E_{1}}+\boldsymbol{T}_{E_{2}}$ and $\boldsymbol{T}_{E_{1}} \perp \boldsymbol{T}_{E_{2}}$. By Lemma 1, $\boldsymbol{T}_{E}=\boldsymbol{T}_{E_{1}+E_{2}}$ holds First, we assert the existence of $P \in A(G)$ such that $P<E_{1}+E_{2}$ and $P \pm E_{i}$ for each $i \in N_{2}$; i.e. $P \wedge E_{i}=0$. Let $P_{1}$ and $P_{2}$ be atoms of $E_{1}$ and $E_{2}$, respectively. $E_{1} \perp E_{2}$ implies $P_{1} \vee P_{2}=P_{1}+P_{2}$. G being irreducible and modular, there exists $P \in A(G)$ with $P \ll P_{1}+P_{2}$ and $P \neq P_{i}$ for each $i \in N_{2}$. Orthomodularity of $G$ insures the existence of $Q \in A(G)$ such that $Q \perp P$ and $P+Q=P_{1}+P_{2}$.

$P \pm E_{\imath}$ or $Q \pm E_{i}$ for each $i \in N_{2}$ shall now be shown:

(i) $P \leqq E_{1}$ leads to the dichotomy

1. $Q \leqq E_{1}$, which implies $P_{2}=P+Q-P_{1} \in B^{\prime}\left(E_{1}\right) \cap B^{\prime}\left(E_{2}\right)$, a contradiction to $B^{\prime}(E)=B^{\prime}\left(E_{1}\right) \oplus B^{\prime}\left(E_{2}\right)$.

2. $Q \leqq E_{2}$, which implies, because of the uniqueness of the representation of $P+Q$ by components of $B^{\prime}\left(E_{i}\right)$, the contradiction $P=P_{1}, Q=P_{2}$.

So 1 . and 2. have the consequence: $P \leqq E_{1} \Rightarrow Q \leqq E_{l}$.

(ii) $P \leqq E_{2}$ admits only $Q \leqq E_{i}$ by similar arguments as in (i). The discussion for $Q \leqq E_{\imath}$ for each $i \in N_{2}$ is in a completely analogous way so that finally

$$
P \leqq E_{i} \text { or } Q \leqq E_{i} \text { for each } i \in N_{2} .
$$

Without loss of generality let us suppose $P \$ E_{i}$ for each $i \in N_{2}$; i.e. $P \wedge E_{i}=0$. Since $P<E_{1}+E_{2}$, so $\boldsymbol{T}_{E_{1}} P+\boldsymbol{T}_{E_{2}} P=\boldsymbol{T}_{E_{1}+E_{2}} P=P$ and thus $T_{E_{1}} P \leqq P$ for each $i \in N_{2}$. From this there follows $\boldsymbol{T}_{E_{1}} P \leqq P \wedge E_{i}=0$, hence $\boldsymbol{T}_{E_{l}} P=0$ with the contradiction $P=0$.

We can sharpen Theorem 6 by

Theorem 7. If $G$ is irreducible and modular, then no $\boldsymbol{T}_{E} \in \mathscr{T}(G)$ is non-trivially orthodecomposable by idempotents of $\mathscr{T} \subset \mathscr{B}\left(B^{\prime}\right)$. 
Proof. Assume the existence of $\boldsymbol{T}_{E} \neq \boldsymbol{T}_{\mathbf{1}}$ with $\boldsymbol{T}_{E}=\boldsymbol{T}_{1}+\boldsymbol{T}_{2}, \boldsymbol{T}_{1} \boldsymbol{T}_{2}$ $=\boldsymbol{T}_{2} \boldsymbol{T}_{1}=\mathbf{0}, \boldsymbol{T}_{i}^{2}=\boldsymbol{T}_{i} \in \mathscr{T}$ and $\boldsymbol{T}_{i} \neq \mathbf{0}$ for each $i \in \boldsymbol{N}_{2}$.

These assumptions have the immediate consequence $\boldsymbol{T}_{i} F \leqq E$ for all $F \in L$ and each $i \in N_{2}$. Moreover, $\boldsymbol{T}_{i} E<E$ since, otherwise, $\boldsymbol{T}_{1} E=E$ for instance would imply $\boldsymbol{T}_{2} E=0$.

Then, because of $0 \leqq \boldsymbol{T}_{2} F \leqq E$ for all $F \in L, \boldsymbol{T}_{2}^{2}=\boldsymbol{T}_{2}$ implies $\boldsymbol{T}_{2} F=0$ for all $F \in L$, hence $\boldsymbol{T}_{2}=\mathbf{0}$ contrary to $\boldsymbol{T}_{i} \neq \mathbf{0}$ for each $i \in \boldsymbol{N}_{2}$. An analogous argument excludes $\boldsymbol{T}_{2} E=E$. From $\boldsymbol{T}_{i} E<E$ for each $i \in \boldsymbol{N}_{2}$, there follows $K_{0}(E) \subset K_{0}\left(\boldsymbol{T}_{i} E\right)$. Determining $E_{i} \in G$ for each $i \in N_{2}$ by $K_{0}\left(\boldsymbol{T}_{i} E\right)=K_{0}\left(E_{i}\right)$ (see [3]), we get $E_{i}<E . \boldsymbol{T}_{i} E \leqq E_{i}<E$ has the consequence $\boldsymbol{T}_{i} E \leqq \boldsymbol{T}_{i} E_{i}$ $\leqq \boldsymbol{T}_{i} E$, hence $\boldsymbol{T}_{i} E_{i}=\boldsymbol{T}_{i} E$. To show $\boldsymbol{T}_{i} E_{i}=E_{i}, \boldsymbol{T}_{i} E_{i}<E_{i}$ must be excluded, for $T_{E_{1}} E_{i} \leqq E_{i}$ holds by construction of $E_{i}$. Assume $T_{i} E_{i}<E_{i}$ : then, by Lemma 7 in [2], there exists $V_{i} \in K_{1}\left(E_{i}\right)$ such that $\left\langle V_{i}, \boldsymbol{T}_{i} E_{i}\right\rangle\left\langle\left\langle V_{l}, E_{i}\right\rangle=1\right.$. Therefore, $K_{1}\left(\boldsymbol{T}_{i} E_{i}\right) \subset K_{1}\left(E_{i}\right)$, and orthomodularity of $W$ gives $K_{1}\left(E_{i}\right)$ $=K_{1}\left(\boldsymbol{T}_{i} E_{i}\right) \vee\left(K_{1}\left(E_{i}\right) \wedge K_{0}\left(\boldsymbol{T}_{i} E_{i}\right)\right)=K_{1}\left(\boldsymbol{T}_{i} E_{i}\right) \vee\left(K_{1}\left(E_{i}\right) \wedge K_{0}\left(E_{i}\right)\right)$, whence the contradiction $K_{1}\left(E_{i}\right) \wedge K_{0}\left(E_{i}\right) \neq \emptyset$.

So $\boldsymbol{T}_{i} E_{i}=E_{i}$ for each $i \in N_{2}$ holds and thus $E=E_{1}+E_{2}$ and $\boldsymbol{T}_{E_{1}} \perp \boldsymbol{T}_{E_{2}}$. Any $F_{i} \in \operatorname{Im} \boldsymbol{T}_{i}$ satisfies $F_{i}<E$, i.e. $F_{i}=\boldsymbol{T}_{i} F_{i} \leqq \boldsymbol{T}_{i} E=\boldsymbol{T}_{i} E_{i}=E_{i}<E$. Consequently, $F_{i} \in L_{0} K_{0}\left(E_{i}\right) \subset \operatorname{Im} \boldsymbol{T}_{E_{i}}$, which implies $\boldsymbol{T}_{E_{i}} \boldsymbol{T}_{i}=\boldsymbol{T}_{i}$. Since $\boldsymbol{T}_{E}=\boldsymbol{T}_{1}+\boldsymbol{T}_{2}$, so $\boldsymbol{T}_{E_{1}}=\boldsymbol{T}_{E_{1}} \boldsymbol{T}_{E}=\boldsymbol{T}_{E_{1}} \boldsymbol{T}_{1}+\boldsymbol{T}_{E_{1}} \boldsymbol{T}_{2}$. In particular, $\boldsymbol{T}_{E_{1}}=\boldsymbol{T}_{1}$ $+\boldsymbol{T}_{E_{1}} \boldsymbol{T}_{2}$ and $\boldsymbol{T}_{E_{2}}=\boldsymbol{T}_{E_{2}} \boldsymbol{T}_{1}+\boldsymbol{T}_{2}$. Multiplying these equations by $\boldsymbol{T}_{E_{2}}$ and $\boldsymbol{T}_{E_{1}}$, respectively, and using $\boldsymbol{T}_{E_{1}} \perp \boldsymbol{T}_{E_{2}}$ give $\boldsymbol{T}_{E_{2}} \boldsymbol{T}_{1}=\boldsymbol{T}_{E_{1}} \boldsymbol{T}_{2}=\mathbf{0}$. So finally, $\boldsymbol{T}_{E_{2}}=\boldsymbol{T}_{i}$ for each $i \in \boldsymbol{N}_{2}$ and thus $\boldsymbol{T}_{E}=\boldsymbol{T}_{E_{1}}+\boldsymbol{T}_{E_{2}}$ contrary to Theorem 6 .

We conclude this section by a statement on chains in $\mathscr{T}(G)$.

Theorem 8. Any chain in $\mathscr{T}(G)$ is linearly independent.

Proof. It suffices to consider only proper chains in $\mathscr{T}(G)$. They are finite, since $B^{\prime}$ is finite-dimensional. Let $\left(\boldsymbol{T}_{E_{2}}\right)_{i \in N_{n}}$ be an ascending finite chain with $n \in \boldsymbol{N}$ and suppose $\sum_{i \in \boldsymbol{N}_{n}} \beta_{i} \boldsymbol{T}_{E_{i}}=\mathbf{0}, \beta_{i} \in \boldsymbol{R}$. Orthomodularity of $\mathscr{T}(G)$ implies $\boldsymbol{T}_{E_{n}}=\boldsymbol{T}_{E_{n-1}} \vee \boldsymbol{T}_{E_{n} \wedge E_{n-1}^{\natural}}$ whence, by the chain property, $\boldsymbol{T}_{E_{i}} \perp \boldsymbol{T}_{E_{n} \wedge E_{n-1}^{\dagger}}$ for all $i \in \boldsymbol{N}_{n-1}$. Applying Theorem 13 and its Corollary 1 of [2], we obtain

$$
\boldsymbol{T}_{E_{n} \wedge E_{n-1}^{\perp}} \sum_{i \in \boldsymbol{N}_{n}} \beta_{i} \boldsymbol{T}_{E_{l}}=\beta_{n} \boldsymbol{T}_{E_{n} \wedge E_{n-1}^{\dagger}} \boldsymbol{T}_{E_{n}}=\beta_{n} \boldsymbol{T}_{E_{n} \wedge E_{n-1}^{\dagger}}=\mathbf{0} .
$$

From $\left(\boldsymbol{T}_{E_{l}}\right)_{i \in \boldsymbol{N}_{n}}$ being a proper chain there follows $\beta_{n}=0$. The same procedure applied to $\boldsymbol{T}_{E_{n-1}}$ verifies the assertion by recursion.

\section{The Algebra $\mathscr{B}\left(B^{\prime}\right)$}

Remark 2. Theorem 21 in [2] is incorrectly formulated. Its correct version is: "If $G$ is irreducible, then $\mathscr{A}(G)=\mathscr{B}\left(B^{\prime}\right)$ ". This means that, if $G$ is irreducible, there exist no invariant subspaces of $B^{\prime}$ except $(0)$ and $B^{\prime}$ for the $\boldsymbol{R}$-algebra $\mathscr{A}(G)$ generated by $\mathscr{T}(G)$ (see [2]). In this case $\mathscr{A}(G)$ is 
called strictly irreducible [6]. The correction consists in only substituting $\mathscr{A}(G)$ for $\mathscr{A}$ in the Theorems 20 and 21 of [2]. However since we had only outlined the proof of Theorem 21, a complete proof is thought to be necessary. The proof of Theorem 20 shows that the commutant $\mathscr{A}(G)^{\prime}$ of $\mathscr{A}(G)$ is isomorphic with the reals $\boldsymbol{R}$, which is a sharpening of Schur's lemma.

Definition 2 [6]. (i) An algebra $\mathscr{A}$ of linear operators over a vector space $\mathscr{X}$ is said to be $k$-fold transitive on $\mathscr{X}$ iff for any $k$ linearly independent $x_{i} \in \mathscr{X}\left(i \in \boldsymbol{N}_{k}\right)$ and $k y_{i} \in \mathscr{X}$ there exists $\boldsymbol{T} \in \mathscr{A}$ such that $\boldsymbol{T} x_{i}=y_{i}$ holds.

(ii) $\mathscr{A}$ is called strictly dense on $\mathscr{X}$ iff $\mathscr{A}$ is $k$-fold transitive for any $k \in N$.

Remark 3. (i) 1 -fold transitivity is equivalent to irreducibility.

(ii) If $\mathscr{A}$ is 2 -fold transitive, then $\mathscr{A}$ is already strictly dense ([6], Lemma (2.4.3)).

Definition 3 [1]. Let $M$ be an $\mathscr{A}$-module.

(i) $M$ is said to be faithful iff $a M \neq(0)$ for every $a \in \mathscr{A} \backslash\{0\}$.

(ii) $M$ is said to be irreducible iff $M$ and (0) are the only $\mathscr{A}$-submodules and $\mathscr{A} M=\left\{\sum_{i \in \boldsymbol{N}_{n}} a_{i} m_{l} \mid a_{i} \in \mathscr{A}\right.$ and $\left.m_{l} \in M, n \in \boldsymbol{N}\right\} \neq(0)$ holds.

Definition 4 [1]. A ring $\mathscr{A}$ is said to be primitive iff there exists a faithful irreducible $\mathscr{A}$-module $M$.

So we are prepared for the formulation of Jacobson's density theorem [1]: If $\mathscr{A}^{\prime}=\operatorname{Hom}_{\mathscr{A}}(M, M)$ is the centralizer of a faithful irreducible $\mathscr{A}$-module $M$, then $\mathscr{A}$ is strictly dense in $\operatorname{Hom}_{\mathscr{A}}(M, M)$. $\left(\mathscr{A}^{\prime}\right.$ is, by Schur's lemma, a field!)

Remark 4. Concerning $B^{\prime}$ we observe that

1. $B^{\prime}$ as $\mathscr{A}(G)$-module is faithful for $\mathscr{A}(G) \subseteq \mathscr{B}\left(B^{\prime}\right)$.

2. $G$ irreducible implies $B^{\prime}$ is irreducible for $\mathscr{A}(G)$, which is, since $\operatorname{dim} B^{\prime}=N<\infty$, equivalent to the strict irreducibility of $B^{\prime}$ for $\mathscr{A}(G)$.

3. $\mathscr{A}(G)^{\prime}$ is isomorphic to $\boldsymbol{R}$, thus $\mathscr{A}(G)^{\prime \prime}=\mathscr{B}\left(B^{\prime}\right)$. We have only to prove $\mathscr{A}(G)=\mathscr{A}(G)^{\prime \prime}$ and Theorem 21 in [2] will then be verified in detail:

By the above density theorem $\mathscr{A}(G)$ is strictly dense in $\mathscr{A}(G)^{\prime \prime}=\mathscr{B}\left(B^{\prime}\right)$. For any $\boldsymbol{T} \in \mathscr{B}\left(B^{\prime}\right)$ and any basis $\left\{Y_{i} \mid i \in \boldsymbol{N}_{N}\right\}$ of $B^{\prime}$ we define $\boldsymbol{T} Y_{i}=\bar{Y}_{i}$ for each $i \in N_{N}$. Since $\mathscr{A}(G)$ is dense, there exists $A \in \mathscr{A}(G)$ such that $\boldsymbol{A} Y_{i}=\bar{Y}_{i}$. Therefore $\boldsymbol{T}$ and $\boldsymbol{A}$ coincide on the basis choosen, hence $\boldsymbol{T}=\boldsymbol{A}$. This proves $\mathscr{B}\left(B^{\prime}\right) \subseteq \mathscr{A}(G)$ and completes the proof of Theorem 21 in [2].

Remark 5. Using Mielnik's terminology [4], we see that the set of all physical filters $\boldsymbol{T}_{E}$ determines the $\boldsymbol{R}$-algebra of all linear operators of $B^{\prime}$ which is generated by the set of all physical decision effects $E$.

Let $\mathscr{L}(\mathscr{S})$ and $\mathscr{R}(\mathscr{S})$ denote the left and right annihilator, respectively, for any $\mathscr{S} \subseteq \mathscr{B}\left(B^{\prime}\right)$. Being the algebra of all linear operators over $B^{\prime}$, $\mathscr{B}\left(B^{\prime}\right)$ satisfies 
1. $\mathscr{L}(\mathscr{S})=\mathscr{B}\left(B^{\prime}\right) \boldsymbol{I}_{1}, \quad$ 2. $\mathscr{R}(\mathscr{S})=\boldsymbol{I}_{2} \mathscr{B}\left(B^{\prime}\right)$

for all $\mathscr{S} \subseteq \mathscr{B}\left(B^{\prime}\right)$ and $\boldsymbol{I}_{1}, \boldsymbol{I}_{2}$ idempotents of $\mathscr{B}\left(B^{\prime}\right)$.

1. and 2. are the defining properties of a Baer-ring. By Remark $5 \mathscr{A}(G)$ is such a ring and there even holds that $\mathscr{B}\left(B^{\prime}\right)$, being a Banach algebra, is an annihilator algebra, which can be inferred from a theorem by Rickart [6]:

1. $\mathscr{B}(\mathscr{X})$ being an annihilator Banach algebra is equivalent with the Banach space $\mathscr{X}$ being reflexive. From the textbook [6] we need 4 other theorems:

2. For each minimal right ideal $\mathscr{R}$ of an algebra $\mathscr{A}$ with $\mathscr{R}^{2} \neq\{0\}$ there exists an idempotent $e \in \mathscr{A}$ such that $\mathscr{R}=e \mathscr{A}$ and $e \mathscr{A} e$ is a field with unit element $e$ (this $e$ is minimal!).

3. For each minimal idempotent $e \in \mathscr{A} \in \mathscr{A}$ is a minimal right ideal, A e a minimal left ideal.

4. For each minimal idempotent of a Banach algebra there holds

(i) e.Ae is isomorphic either to the reals or the complexes or the quaternions.

(ii) If $\mathscr{A}$ is complex, $e \mathscr{A} e=e$.

5. In a semisimple annihilator algebra is $(0)$ the only right ideal which contains no minimal right ideals.

Consequence. By Theorem 20 in [2] $\mathscr{A}(G)=\mathscr{B}\left(B^{\prime}\right)$ is simple, hence minimal idempotents exist and 2.-5. give a biunivocal correspondence between the minimal idempotents and the minimal right (and left) ideals (respectively).

We are now prepared for an investigation of all minimal idempotents (and hence all minimal right ideals) in $\mathscr{B}\left(B^{\prime}\right)$ : Reflexity of $B$ and $B^{\prime}$ implies the canonical isomorphisms: $B^{\prime} \otimes_{\boldsymbol{R}} B=\mathscr{B}(B) ; B \otimes_{\boldsymbol{R}} B^{\prime}=\mathscr{B}\left(B^{\prime}\right)$. So we may define the following linear operators over $B$ and $B^{\prime}$, respectively:

$$
\begin{aligned}
& (Y \otimes X) \bar{X}=\langle\bar{X}, Y\rangle X \quad \text { for all } \bar{X} \in B \quad \text { and any } \quad Y \in B^{\prime}, X \in B \text {; } \\
& (X \otimes Y) \bar{Y}=\langle X, \bar{Y}\rangle Y \quad \text { for all } \bar{Y} \in B^{\prime} \quad \text { and any } \quad X \in B, Y \in B^{\prime} .
\end{aligned}
$$

Let $X \otimes Y$ be positive, i.e. $(X \otimes Y)\left[B_{+}^{\prime}\right] \subseteq B_{+}^{\prime}$, then $(X \otimes Y) \bar{Y} \in B_{+}^{\prime}$ for all $\bar{Y} \in B_{+}^{\prime}$. There are two cases to be distinguished:

(i) $Y \in-B_{+}^{\prime}$ implies $\langle X, \bar{Y}\rangle \leqq 0$ for all $\bar{Y} \in B_{+}^{\prime}$, thus $X \in-B_{+}$.

(ii) $Y \in B_{+}^{\prime}$ implies $\langle X, \bar{Y}\rangle \geqq 0$ for all $\bar{Y} \in B_{+}^{\prime}$ thus $X \in B_{+}$. Summerizing we can state

Theorem 9. $\mathscr{T}_{1}:=\left\{X \otimes Y \mid X \in B_{+}\right.$and $\left.Y \in B_{+}^{\prime}\right\}$ is the set of all positive operators of rank 1 in $\mathscr{B}\left(B^{\prime}\right)$. 
Lemma 2. Every idempotent $\boldsymbol{I} \in \mathscr{B}\left(B^{\prime}\right)$ with rank 1 is minimal.

Proof. Assume $\boldsymbol{I}=\boldsymbol{I}_{1}+\boldsymbol{I}_{2}$ such that $\boldsymbol{I}_{1} \boldsymbol{I}_{2}=\boldsymbol{I}_{2} \boldsymbol{I}_{1}=\mathbf{0}$ and $I_{i} \neq \mathbf{0}$ for each $i \in N_{2}$. Then we have $\operatorname{Im} I=\operatorname{Im} I_{1} \oplus \operatorname{Im} I_{2}$, hence $\operatorname{dim} \operatorname{Im} I$ $=\operatorname{dim} \operatorname{Im} I_{1}+\operatorname{dim} \operatorname{Im} I_{2} \geqq 2$, contrary to $\operatorname{dim} \operatorname{Im} I=1$.

Theorem 10. Every positive minimal idempotent $I \in \mathscr{B}\left(B^{\prime}\right)$ has the representation

$$
X \otimes Y \quad \text { with }\langle X, Y\rangle=1, X \in B_{+} \text {and } Y \in B_{+}^{\prime} .
$$

Proof. According to [6], p. 65, a right ideal of $\mathscr{B}\left(B^{\prime}\right)$ is minimal iff it consists of elements of rank 1. From Theorem 9 and Lemma 2 there follows for such an idempotent $\boldsymbol{I}$ that $\boldsymbol{I}=X \otimes Y, X \in B_{+}, Y \in B_{+}^{\prime}$ and idempotence of $\boldsymbol{I}$ requires

$$
(X \otimes Y)^{2} \bar{Y}=\langle X, Y\rangle\langle X, \bar{Y}\rangle Y=\langle X, Y\rangle(X \otimes Y) \bar{Y}=(X \otimes Y) \bar{Y}
$$

for all $\bar{Y} \in B^{\prime}$. This is satisfied iff $\langle X, Y\rangle=1$.

2. and 5. of Section II admit the representations: every $X \in B_{+}$can be written as $X=\alpha V, \alpha \in \boldsymbol{R}_{+}$and $V \in K$; every $Y \in B_{+}^{\prime}$ can be written as $Y=\beta F, \beta \in \boldsymbol{R}_{+}$and $F \in L$. Therefore, any $\boldsymbol{I}$ from Theorem 10 can be represented as $I=\alpha \beta V \otimes F$ with $\beta\langle V, F\rangle=1$. If $Y=\beta F$ then $\beta$ can be choosen so that $K_{1}(F) \neq \emptyset$ because $K$ is compact. This leads us to

Theorem 11. Every positive minimal idempotent $I \in \mathscr{B}\left(B^{\prime}\right)$ can be represented by $\boldsymbol{I}=\beta V \otimes F$ with $\beta\langle V, F\rangle=1$ and $K_{1}(F) \neq \emptyset$.

Corollary. $V \otimes F$ is a minimal idempotent iff $V \in K_{1}(F)$. As an important consequence of Theorem 11 we may verify

Theorem 12. Every atom $\boldsymbol{T}_{\boldsymbol{P}} \in \mathscr{T}(G)$ (i.e. $\boldsymbol{T}_{\boldsymbol{P}} \in A \mathscr{T}(G)$ ) is a positive minimal idempotent satisfying

$$
\boldsymbol{T}_{P}=V_{P} \otimes P, P \in A(G) \quad \text { and } \quad\left\{V_{P}\right\}=K_{1}(P) .
$$

Proof. Per definitionem of $\mathscr{T}(G) \boldsymbol{T}_{P}$ is idempotent and operates by reason of the preface to Theorem 20 in [2] as $T_{P} Y=\left\langle V_{P}, Y\right\rangle P$ for all $Y \in B^{\prime}$, thus $T_{P}=V_{P} \otimes P$.

From 4. of the quoted theorems in Rickart's textbook [6] there follows in particular that each $\boldsymbol{T}_{P} \in A \mathscr{T}(G)$ satisfies $\boldsymbol{T}_{\boldsymbol{P}} \mathscr{B}\left(B^{\prime}\right) \boldsymbol{T}_{P}=\boldsymbol{R} \boldsymbol{T}_{\boldsymbol{P}}$ $=T_{P} R$. Of course, we would have been able to calculate this equality directly, ignoring, however, its connexion with irreducibility of idempotents in $\mathscr{B}\left(B^{\prime}\right)$.

The next step leads to a linear order isomorphism between $B, B^{\prime}$ and minimal ideals of $\mathscr{B}\left(B^{\prime}\right)$. To this purpose we show 
Theorem 13. For arbitrary but fixed $P \in A(G)$ the sets

$$
\mathscr{R}_{P}:=\{X \otimes P \mid X \in B\} \text { and } \mathscr{L}_{P}:=\left\{V_{P} \otimes Y \mid Y \in B^{\prime}\right\}
$$

are minimal right and left ideals, respectively, satisfying

$$
\mathscr{R}_{P}=\boldsymbol{T}_{P} \mathscr{B}\left(B^{\prime}\right), \mathscr{L}_{P}=\mathscr{B}\left(B^{\prime}\right) \boldsymbol{T}_{P} .
$$

Proof. The ideal property is obvious. Besides, each element of the ideals has rank 1 . We have only to prove $\mathscr{L}_{P}=\mathscr{B}\left(B^{\prime}\right) \boldsymbol{T}_{P}$, the verification of $\mathscr{R}_{P}=\boldsymbol{T}_{P} \mathscr{B}\left(B^{\prime}\right)$ is then in a completely analogous way and will be omitted. Let $\left\{X_{i} \mid i \in N_{N}\right\}$ and $\left\{Y_{j} \mid j \in N_{N}\right\}$ be bases of $B$ and $B^{\prime}$, respectively. Then every $\boldsymbol{T} \in \mathscr{B}\left(B^{\prime}\right)$ admits the representation $\boldsymbol{T}=\sum_{i, j \in \boldsymbol{N}_{N}} t_{i j} X_{i} \otimes Y_{j}$; thus, for all $Y \in B^{\prime}$, there holds $\boldsymbol{T}_{\boldsymbol{P}} Y=\boldsymbol{T}\left(V_{\boldsymbol{P}} \otimes P\right) Y=\sum_{i, j \in \boldsymbol{N}_{N}} t_{i j}\left\langle X_{i}, P\right\rangle$ $\cdot\left(V_{P} \otimes Y_{j}\right) Y$, whence, with $Y:=\sum_{i, j \in N_{N}} t_{i j}\left\langle X_{i}, P\right\rangle Y_{j}, \boldsymbol{T} V_{P} \otimes P=V_{P} \otimes Y \in \mathscr{L}_{P}$, i.e. $\mathscr{B}\left(B^{\prime}\right) \boldsymbol{T} \leqq \mathscr{L}_{P}$. Consequently, for every $V_{P} \otimes Y \in \mathscr{L}_{P}:\left(V_{P} \otimes Y\right)\left(V_{P} \otimes P\right)$ $=\left\langle V_{P}, \cdot\right\rangle\left\langle V_{P}, P\right\rangle Y=\left\langle V_{P}, \cdot\right\rangle Y=V_{P} \otimes Y$ since $\left\langle V_{P}, P\right\rangle=1$. Hence $\mathscr{L}_{P} \cong \mathscr{B}\left(B^{\prime}\right) T_{P}$.

Corollary. The correspondences $B \rightarrow \mathscr{R}_{P}$ and $B^{\prime} \rightarrow \mathscr{L}_{P}$ are linear bijections.

4. of the Rickart-theorems quoted yields $\mathscr{R}_{P} \mathscr{L}_{P}=\boldsymbol{T}_{P} \mathscr{B}\left(B^{\prime}\right) \mathscr{B}\left(B^{\prime}\right) \boldsymbol{T}_{P}$ $=\boldsymbol{T}_{P} \mathscr{B}\left(B^{\prime}\right) \boldsymbol{T}_{P}=\boldsymbol{R} \boldsymbol{T}_{P}$, which leads because of $(X \otimes P)\left(V_{P} \otimes Y\right)=\langle X, Y\rangle \boldsymbol{T}_{P}$ to the existence of a bilinear functional $\Gamma$ over $\mathscr{R}_{P} \times \mathscr{L}_{P}$ given by $\Gamma\left(X \otimes P, V_{P} \otimes Y\right)=\langle X, Y\rangle=\mu(X, Y) . \quad \Gamma \quad$ is, therefore, the canonical bilinear functional over $\mathscr{R}_{P} \times \mathscr{L}_{P}$, because $\langle\cdot, \cdot\rangle$ has this property on $B \times B^{\prime}$. This enables us to formulate

Theorem 14. (i) The bijections from the preceding corollary are order isomorphisms.

(ii) The sets $L \subset B^{\prime}, G \subset B^{\prime}, K \subset B$ are represented by

$\hat{\mathscr{L}}_{P}=\left\{V_{P} \otimes F \mid F \in L\right\}, \quad \mathscr{G}_{P}=\left\{V_{P} \otimes E \mid E \in G\right\}$ and $\mathscr{K}_{P}=\left\{V_{P} \otimes P \mid V \in K\right\}$, respectively.

Remark 6. According to Theorem 10 any minimal idempotent $X_{0} \otimes Y_{0}$ with $\left\langle X_{0}, Y_{0}\right\rangle=1$ can be substituted for $\boldsymbol{T}_{\boldsymbol{P}}$ in the preceding theorem, for it leads to equivalent representations.

Open remains the question of what algebras posses a bilinear functional $\Gamma$ satisfying all postulates of $\mu$ (and its extension) and of what subsets of these algebras represent $L \subset B^{\prime}, G \subset B^{\prime}$ and $K \subset B$, i.e. satisfy all the axioms postulated of $L$ and $K$. Theorem 14 suggests to attempt a characterization of the dual pair $\left(B, B^{\prime}\right)$ by the algebra $\mathscr{B}\left(B^{\prime}\right)$. The following considerations prepare this task. 
Lemma 3. In $\left(\mathscr{B}\left(B^{\prime}\right),\|\cdot\|_{b}, N\right)$ as a Banach space there exists an additional norm defined by $\|\boldsymbol{T}\|_{L}:=\sup \{\|\boldsymbol{T} F\| \mid F \in L\}, b^{N}$ denoting the $N$-dimensional unit ball of $B^{\prime}$.

Proof. Verification of the norm axioms: 1) $\|\boldsymbol{T}\|_{L} \geqq 0$ for all $\boldsymbol{T} \in \mathscr{B}\left(B^{\prime}\right)$. 2) $\|\boldsymbol{T}\|_{L}=0$ implies $\|\boldsymbol{T} F\|=0$ for all $F \in L$, hence $\boldsymbol{T} F=0$ for all $F \in L$. Since $L$ is generating in $B^{\prime}$, so $\boldsymbol{T}=\mathbf{0}$. Immediately from the above definition there follow

3. $\|\beta \boldsymbol{T}\|_{L}=|\beta|\|\boldsymbol{T}\|_{L}$ for all $\beta \in \boldsymbol{R}$ and

4. $\left\|\boldsymbol{T}_{1}+\boldsymbol{T}_{2}\right\|_{L} \leqq\left\|\boldsymbol{T}_{1}\right\|_{L}+\left\|\boldsymbol{T}_{2}\right\|_{L}$.

Remark 7. Concerning this norm $\mathscr{B}\left(B^{\prime}\right)$ is as a finite-dimensional vector space complete and the two norms $\|\cdot\|_{b^{\prime}} N$ and $\|\cdot\|_{L}$ determine the same topology in $\mathscr{B}\left(B^{\prime}\right)$, hence they are equivalent. Therefore, all $\boldsymbol{T} \in \mathscr{B}\left(B^{\prime}\right)$ are already distinguished sufficiently sharp by the effects $F \in L$. This is the physical meaning of the norm $\|\cdot\|_{L}$.

We can even strengthen Lemma 3 by

Theorem 15. $\left(\mathscr{B}\left(B^{\prime}\right),\|\cdot\|_{L}\right)$ is (just as $\left.\left(\mathscr{B}\left(B^{\prime}\right),\|\cdot\|_{b^{\prime}} N\right)\right)$ a Banach algebra.

Proof. Since $\|\boldsymbol{T} F\| \leqq \beta_{L}\|F\|$ with $\beta_{L} \in \boldsymbol{R}_{+}^{*}$ for all $F \in L$, we have in addition to the norm properties in Lemma 3 ,

$$
\left\|\boldsymbol{T}_{1} \boldsymbol{T}_{2}\right\|_{L}=\sup \left\{\left\|\boldsymbol{T}_{1} \boldsymbol{T}_{2} F\right\| \mid F \in L\right\} \leqq\left\|\boldsymbol{T}_{1}\right\|_{L}\left\|\boldsymbol{T}_{2} F\right\| \leqq\left\|\boldsymbol{T}_{1}\right\|_{L}\left\|\boldsymbol{T}_{2}\right\|_{L} .
$$

Moreover, there holds $\left\|\boldsymbol{T}_{\mathbf{1}}\right\|_{L}=\left\|\boldsymbol{i d}_{B^{\prime}}\right\|_{L}=\sup \{\|F\| \mid F \in L\}=1$.

We intend now to tackle the problem of how to make $\left(\mathscr{B}\left(B^{\prime}\right),\|\cdot\|_{L}\right)$ a *-algebra with $\mathscr{T}(G)$ as a subset of the set of all elements remaining fixed under the involution $*$. Remark 5 gives an appropriate hint:

$\mathscr{A}(G)$ is the smallest $\boldsymbol{R}$-algebra containing $\mathscr{T}(G)$. Thus it contains the $\boldsymbol{R}$-algebra of all finite linear combinations of all finite products of elements of $\mathscr{T}(G)$. By Remark 5 it equals $\mathscr{B}\left(B^{\prime}\right)$. Consequently, each $\boldsymbol{T} \in \mathscr{B}\left(B^{\prime}\right)$ has a representation by finitely many $\boldsymbol{T}_{E} \in \mathscr{T}(G)$ because of $\operatorname{dim} \mathscr{B}\left(B^{\prime}\right)=N^{2}<\infty$. Without loss of generality these $\boldsymbol{T}_{E}$ may be selected to be linearly independent:

$$
\begin{gathered}
\boldsymbol{T}=\sum_{i_{1} \in \boldsymbol{N}_{l}} t\left(i_{1}\right) \boldsymbol{T}_{E_{u_{1}}}+\sum_{\substack{i_{1}, i_{2} \\
i_{1} \neq i_{2}}} t\left(i_{1} i_{2}\right) \boldsymbol{T}_{E_{l_{1}}} \boldsymbol{T}_{E_{l_{2}}}+\cdots \\
\cdots+\sum_{\substack{i_{1}, \ldots, i_{m} \\
i_{1} \neq i_{2} \neq \cdots \neq i_{m}}} t\left(i_{1}, \ldots, i_{m}\right) \boldsymbol{T}_{E_{t_{1}}} \boldsymbol{T}_{E_{l_{2}}} \ldots \boldsymbol{T}_{E_{l_{m}}} ;
\end{gathered}
$$

$m \in N, i_{k} \in N_{l}$ for all $k \in N_{m}$, where $l \leqq \operatorname{dim} \mathscr{B}\left(B^{\prime}\right)=N^{2}$.

We define for every $m \in N$ :

$$
\mathscr{T}(G)^{m}:=\left\{\boldsymbol{T}_{E_{t_{l}}} \boldsymbol{T}_{E_{t_{2}}} \ldots \boldsymbol{T}_{E_{l_{m}}} \mid \boldsymbol{T}_{E_{t_{k}}} \in \mathscr{T}(G) \text { and } k \in \boldsymbol{N}_{m}\right\} .
$$


Then we regard on $\bigcup_{m \in \mathbf{N}} \mathscr{T}(G)^{m}$ the relation * defined by

$$
\left(\boldsymbol{T}_{E_{u_{1}}} \boldsymbol{T}_{E_{v_{2}}} \ldots \boldsymbol{T}_{E_{l_{m-1}}} \boldsymbol{T}_{E_{l_{m}}}\right)^{*}=\boldsymbol{T}_{E_{l_{m}}} \boldsymbol{T}_{E_{l_{m-1}}} \ldots \boldsymbol{T}_{E_{t_{2}}} \boldsymbol{T}_{E_{2_{1}}} .
$$

This confronts us with the question when the defining property for * to be a mapping holds: "For any $\boldsymbol{T}_{1}, \boldsymbol{T}_{2} \in \mathscr{T}(G)^{m}: \boldsymbol{T}_{1}=\boldsymbol{T}_{2} \Rightarrow \boldsymbol{T}_{1}^{*}=\boldsymbol{T}_{2}^{*}$. Since $\boldsymbol{T}_{E}=\boldsymbol{T}_{E} \boldsymbol{T}_{E}$ implies $\boldsymbol{T}_{E}^{*}=\boldsymbol{T}_{E}$ for each $\boldsymbol{T}_{E} \in \mathscr{T}(G)$ the defining property of $*$ is obviously valid for all $\boldsymbol{T}_{E} \in \mathscr{T}(G)$ being a subset of the set of all fixed elements under *. Let us consider $\mathscr{T}(G)^{2}$ and assume $\boldsymbol{T}_{E_{1}} \boldsymbol{T}_{E_{2}}=\boldsymbol{T}_{E_{3}} \boldsymbol{T}_{E_{4}}$. The question is whether " $\boldsymbol{T}_{E_{1}} \boldsymbol{T}_{E_{2}}=\boldsymbol{T}_{E_{3}} \boldsymbol{T}_{E_{4}}$ implies $\boldsymbol{T}_{E_{2}} \boldsymbol{T}_{E_{1}}=\boldsymbol{T}_{E_{4}} \boldsymbol{T}_{E_{3}}$ " holds.

Theorem 16. For all $i \in N_{4}$ and $\boldsymbol{T}_{E_{1}} \in \mathscr{T}(G): \boldsymbol{T}_{E_{1}} \boldsymbol{T}_{E_{2}}=\boldsymbol{T}_{E_{3}} \boldsymbol{T}_{E_{4}}$ and $E_{1} \mathscr{C} E_{2}$ implies $\boldsymbol{T}_{E_{2}} \boldsymbol{T}_{E_{1}}=\boldsymbol{T}_{E_{4}} \boldsymbol{T}_{E_{3}}$.

Proof. From $\boldsymbol{T}_{E_{1}} \boldsymbol{T}_{E_{2}}=\boldsymbol{T}_{E_{3}} \boldsymbol{T}_{E_{4}}$ there follows $\boldsymbol{T}_{E_{1}} \boldsymbol{T}_{E_{2}}\left(E_{1} \wedge E_{2}\right)=E_{1} \wedge E_{2}$ $=\boldsymbol{T}_{E_{3}} \boldsymbol{T}_{E_{4}}\left(E_{1} \wedge E_{2}\right) \leqq E_{1} \wedge E_{3}$ and $\boldsymbol{T}_{E_{1}} \boldsymbol{T}_{E_{2}}\left(E_{3} \wedge E_{4}\right)=\boldsymbol{T}_{E_{3}} \boldsymbol{T}_{E_{4}}\left(E_{3} \wedge E_{4}\right)$ $=E_{3} \wedge E_{4} \leqq E_{1} \wedge E_{3} . E_{1} \mathscr{C} E_{2}$ is, by Theorem 2, equivalent with $\boldsymbol{T}_{E_{1}} \boldsymbol{T}_{E_{2}}$ $=\boldsymbol{T}_{E_{2}} \boldsymbol{T}_{E_{1}}$. Applying then Theorem 1, we obtain $\boldsymbol{T}_{E_{1} \wedge E_{2}}\left(E_{3} \wedge E_{4}\right)$ $=\boldsymbol{T}_{E_{3}} \boldsymbol{T}_{E_{4}}\left(E_{3} \wedge E_{4}\right)=E_{3} \wedge E_{4} \leqq E_{1} \wedge E_{2} . \quad \boldsymbol{T}_{E_{1} \wedge E_{2}} E_{4}^{\perp}=\boldsymbol{T}_{E_{3}} \boldsymbol{T}_{E_{4}} E_{4}^{\perp}=0$ implies $\left\langle V, \boldsymbol{T}_{E_{1} \wedge E_{2}} E_{4}^{\perp}\right\rangle=\left\langle V, E_{4}^{\perp}\right\rangle=0$ for every $V \in K_{1}\left(E_{1} \wedge E_{2}\right)$, hence $K_{1}\left(E_{1} \wedge E_{2}\right) K_{0}\left(E_{4}^{\perp}\right)=K_{1}\left(E_{4}\right), \quad$ thus $\quad E_{1} \wedge E_{2} \leqq E_{4} . \quad \boldsymbol{T}_{E_{3}} \boldsymbol{T}_{E_{1} \wedge E_{2}}$ $=\boldsymbol{T}_{E_{3}} \boldsymbol{T}_{E_{3}} \boldsymbol{T}_{E_{4}}=\mathbf{0}$ implies $\boldsymbol{T}_{E_{3}} E_{1} \wedge E_{2}=0$, i.e. $0=\left\langle V, \boldsymbol{T}_{E_{3}} E_{1} \wedge E_{2}\right\rangle$ $=\left\langle V, E_{1} \wedge E_{2}\right\rangle$ for every $V \in K_{1}\left(E_{3}^{\perp}\right)$, hence $K_{1}\left(E_{3}^{\perp}\right)=K_{0}\left(E_{3}\right) \leqq K_{0}\left(E_{1} \wedge E_{2}\right)$, thus $E_{1} \wedge E_{2} \leqq E_{3}$. This completes the proof of $E_{1} \wedge E_{2}=E_{3} \wedge E_{4}$ from which $\boldsymbol{T}_{E_{1} \wedge E_{2}}=\boldsymbol{T}_{E_{3} \wedge E_{4}}=\boldsymbol{T}_{E_{3}} \boldsymbol{T}_{E_{4}}$ results and thus $\boldsymbol{T}_{E_{3}} \boldsymbol{T}_{E_{4}}=\boldsymbol{T}_{E_{4}} \boldsymbol{T}_{E_{3}}$.

Physically speaking, the above implication was only verified for commensurable decision effects. Generally, however, the above implication is valid for $(A \mathscr{T}(G))^{2}$ :

Theorem 17. For all $\boldsymbol{T}_{P_{1}}, \boldsymbol{T}_{P_{2}}, \boldsymbol{T}_{Q_{1}}, \boldsymbol{T}_{Q_{2}} \in A \mathscr{T}(G): \boldsymbol{T}_{P_{1}} \boldsymbol{T}_{P_{2}}=\boldsymbol{T}_{Q_{1}} \boldsymbol{T}_{Q_{2}}$ implies $\boldsymbol{T}_{P_{2}} \boldsymbol{T}_{P_{1}}=\boldsymbol{T}_{Q_{2}} \boldsymbol{T}_{Q_{1}}$.

Proof. (i) If $\boldsymbol{T}_{P_{1}} \boldsymbol{T}_{P_{2}}=\boldsymbol{T}_{Q_{1}} \boldsymbol{T}_{Q_{2}}=\mathbf{0}$, then $P_{1} \perp P_{2}$ and $Q_{1} \perp Q_{2}$ and the implication is true.

(ii) If $\boldsymbol{T}_{P_{1}} \boldsymbol{T}_{P_{2}} \neq \mathbf{0}$, then $\boldsymbol{T}_{P_{1}} \boldsymbol{T}_{P_{2}}=\left\langle V_{P_{1}}, P_{2}\right\rangle V_{P_{2}} \otimes P_{1}=\left\langle V_{Q_{1}}, Q_{2}\right\rangle V_{Q_{2}} \otimes Q_{1}$ with $\left\langle V_{P_{1}}, P_{2}\right\rangle \neq 0 \neq\left\langle V_{Q_{1}}, Q_{2}\right\rangle$ implies $P_{1}=Q_{1}$. Moreover, for every atom $P \leqq P_{2}^{\perp}$ we obtain $\boldsymbol{T}_{Q_{2}} \boldsymbol{T}_{P}=\mathbf{0}$, hence $P \leqq Q_{2}^{\perp}$. For every atom $Q \leqq Q_{2}^{\perp}$



Suppose * is a mapping, then * is obviously involutory on $\bigcup_{m \in N} \mathscr{T}(G)^{m}$ and the question arises whether * can be linearly extended to $\mathscr{B}\left(B^{\prime}\right)$ to give an algebra involution. A necessary condition for * to have such an extension is:

"For every $\boldsymbol{T} \in \bigcup_{m \in \boldsymbol{N}} \mathscr{T}(G)^{m}: \boldsymbol{T}^{*}=\mathbf{0} \Rightarrow \boldsymbol{T}=\mathbf{0} . "$ 
Obviously, this implication is true if $E_{i_{j}} \perp E_{i_{j-1}}$ for at least one $j \in \boldsymbol{N}_{m} \backslash\{1\}$. Let us consider $\boldsymbol{T}_{E_{11}} \ldots \boldsymbol{T}_{E_{l_{m}}} \in \mathscr{T}(G)^{m}$ such that $\left(\boldsymbol{T}_{E_{t_{1}}} \ldots \boldsymbol{T}_{E_{l_{m}}}\right)^{*}$ $=\boldsymbol{T}_{E_{l_{m}}} \ldots \boldsymbol{T}_{E_{t_{1}}}=0$. This implies $\left\langle V, \boldsymbol{T}_{E_{l_{m}}} \boldsymbol{T}_{E_{l_{m-1}}} \ldots \boldsymbol{T}_{E_{l_{1}}} F\right\rangle=0$ for every $F \in L$ and every $V \in K$; particularly, $\left\langle V, \boldsymbol{T}_{E_{l_{m}}} \boldsymbol{T}_{E_{l_{m}-1}} \ldots \boldsymbol{T}_{E_{u_{1}}} F\right\rangle$ $=\left\langle V, \boldsymbol{T}_{E_{l_{m-1}}} \ldots \boldsymbol{T}_{E_{u_{1}}} F\right\rangle=0$ for every $V \in K_{1}\left(E_{i_{m}}\right)=K_{0}\left(E_{l_{m}}^{\perp}\right)$. Since $\boldsymbol{T}_{E_{l_{m-1}}} \ldots \boldsymbol{T}_{E_{i_{1}}} F \in L_{0} K_{0}\left(E_{i_{m-1}-1}\right)$, so $\boldsymbol{T}_{E_{l_{m-1}}} \ldots \boldsymbol{T}_{E_{u_{1}}} F \in L_{0} K_{0}\left(E_{i_{m-1}}\right) \cap L_{0} K_{0}\left(E_{i_{m}}^{\perp}\right)$ $=L_{0} K_{0}\left(E_{i_{m-1}} \wedge E_{i_{m}}^{\perp}\right)$. We failed to prove the desired implication generally though it holds in an important special case.

Theorem 18. For every $\boldsymbol{T} \in \bigcup_{m \in \boldsymbol{N}} A \mathscr{T}(G)^{m}: \boldsymbol{T}^{*}=\mathbf{0} \Rightarrow \boldsymbol{T}=\mathbf{0}$.

Proof. By the above consideration we get the following dichotomy:

(i) $P_{i_{m-1}} \wedge P_{i_{m}}^{\perp}=P_{i_{m-1}}$, hence $P_{i_{m-1}} \leqq P_{i_{m}}^{\perp}$ and the implication is true.

(ii) $P_{i_{m-1}} \wedge P_{i_{m}}^{\perp}=0$ leads to $\boldsymbol{T}_{P_{l_{m-1}}} \ldots \boldsymbol{T}_{P_{l_{1}}}=\mathbf{0}$. To $\boldsymbol{T}_{P_{l_{m-1}}} \ldots \boldsymbol{T}_{P_{t_{1}}}=\mathbf{0}$

the same procedure applies and we arrive at least at one $j \in N_{m} \backslash\{1\}$ such that $P_{i_{j}} \perp P_{i_{j-1}}$ which states the validity of the implication. (i) and (ii) express the existence of at least two orthogonal atoms being neighbouring factors of the product in question.

There remains the open question whether for instance modularity of $G$ guarantees the existence of an involution ${ }^{*}$ on $\mathscr{B}\left(B^{\prime}\right)$ which makes $\mathscr{B}\left(B^{\prime}\right)$ a $C^{*}$-algebra with $\mathscr{T}(G)$ in the set of all fixed elements under *. The converse of this question (the open question 3 in [2]) will be answered in a subsequent paper in the affirmative.

\section{References}

1. Behrens, E. A.: Algebren, Bd. 97/97a. Mannheim: Bibliogr. Institut 1965.

2. Dähn, G.: Attempt of an axiomatic foundation of quantum mechanics and more general theories. IV. Commun. math. Phys. 9, 192-211 (1968).

3. Ludwig, G.: Attempt of an axiomatic foundation of quantum mechanics and more general theories. III. Commun. math. Phys. 9, 1-12 (1968).

4. Mielnik, B.: Theory of filters. Commun. math. Phys. 15, 1-46 (1969).

5. Nakamura, M.: The permutability in a certain orthocomplemented lattice. Kodai Math. Sem. Rep. 9, 158-160 (1957).

6. Rickart, C.E.: General theory of Banach algebras. Princeton: Van Nostrand 1960.

Günter Dähn

Mathematisches Institut der Universität

D-7400 Tübingen

Brunnenstraße 27

Federal Republic of Germany 